\title{
Novel mechanism of the negative thermal expansion of doped fullerite $\mathrm{C}_{60}$
}

\author{
M.A. Strzhemechny and I.V. Legchenkova \\ B. Verkin Institute for Low Temperature Physics and Engineering of the National Academy of Sciences of Ukraine \\ 47 Lenin Ave., Kharkov 61103, Ukraine \\ E-mail: strzhemechny@ilt.kharkov.ua
}

Received February 9, 2010

\begin{abstract}
A model for an atomic impurity in an octahedral void of fullerite $\mathrm{C}_{60}$ is suggested. The problem is solved in the spherical oscillator approximation, which is appropriate for the larger rare gas atoms. It is shown that such impurities can contribute to the negative thermal expansions at low temperatures and produce a Schottky-like maximum at higher temperatures.

PACS: 81.05.ub Fullerenes and related materials;

61.72.jj Interstitials;

65.40.-b Thermal properties of crystalline solids.
\end{abstract}

Keywords: fullerite, rare gas atoms, negative thermal expansion.

First low-temperature measurements of thermal expansivities $\alpha(T)$ of pure fullerite $\mathrm{C}_{60}$ using dilatometry [1] and $\mathrm{x}$-ray diffractometry [2] did not reveal any deviations from the standard behavior of any crystal. However, highprecision dilatometry group in Kharkov reported [3] that below $3.4 \mathrm{~K}$ the thermal expansivity of a compacted sample of pure $\mathrm{C}_{60}$ is negative. When doped with chemically neutral species (rare gas atoms [4,5] or simpler close-shell molecules like nitrogen [6], methane [7] and oxygen [8]), the $\mathrm{C}_{60}$ solid showed the same, albeit somewhat different, behavior. In some cases, especially when the dopant was a simple molecule, $\alpha(T)$ exhibited a maximum.

The negativity suggests that the underlying mechanism might be related with transitions between some tunneling states. Similar effects in low-temperature thermal expansivity were previously documented by the same group in rare gas crystals with substitution molecular impurities (cf. Aleksandrovskii et al. [9] and references therein). These effects were explained and analyzed on the basis of the idea [10] that transitions between tunneling rotational states could render the expansivity negative at sufficiently low temperatures when the positive phonon related contribution is negligible. The relevant numeric evaluations were based on Devonshire's [11] calculations of the spectrum of a rotor in a field of cubic symmetry.

Since the energy spacing $\Delta E$ between tunneling states increases with increasing volume $V$, the relevant Grüneisen parameter

$$
\gamma=-\frac{\partial \ln \Delta E}{\partial \ln V}
$$

is negative. At low enough temperatures, when the «standard» phonon contribution to the thermal expansivity is small, the net thermal expansion coefficient

$$
\beta=(k / V)\left[\gamma_{\text {tun }} C_{\text {tun }}+\gamma_{\mathrm{ph}} C_{\mathrm{ph}}\right],
$$

with $k$, the Boltzmann constant and $C$, the respective heat capacity contributions, may become negative (considering that both heat capacities $C$ in Eq. (2) are positive). The current explanation [5] of the negative thermal expansivity effect in $\mathrm{C}_{60}$ is based on the assumption that the relevant states are due to tunneling rotations of the fullerene molecules. The other phenomenon, namely, the maxima in the $\alpha(T)$ dependence was ascribed [5] to the so-called polyamorphic transitions between different orientational glass states. It seems rather unlikely that a $\mathrm{C}_{60}$ molecule with its huge mass can tunnel rotate through a barrier approximately $0.3 \mathrm{eV}$ high with a probability of a few Kelvin in appropriate units. Understanding that, Loktev and coauthors $[12,13]$ put forward the idea that the main negative expansivity contribution comes from molecules in irregular areas of the crystal (grain boundaries, dislocations, orientational domain walls [14], etc.), where the relevant energy barriers are considerably reduced.

From the above-stated it follows that the possible role of dopant particles in $\mathrm{C}_{60}$ has been disregarded so far. At least, molecular dopants could easily contribute to both phenomena observed within the above-mentioned approach of Devonshire [11], considering that the symmetry of the voids of both types is octahedral. However, atomic rare 
gas impurity can also produce similar effects in the lowtemperature expansion and to show that is the aim of this paper.

Let us consider a much simpler case of an atom (rather than a molecule, however simple) placed inside an octahedral void of the face-centered cubic lattice of fullerite $\mathrm{C}_{60}$. Even though the surrounding molecules are shifted due to the presence of the dopant particle, the symmetry of the resulting crystal field felt by the dopant remains predominantly cubic. This crystal field $U(\mathbf{R})$ as a function of the radius vector $\mathbf{R}$ of the center of mass of the dopant particle, referred to the center of the octahedral cavity, can be represented by the function

$$
U(\mathbf{R})=\sum_{N} V_{N}(R) I_{N}(\mathbf{n})
$$

where $\mathbf{R} \equiv R \mathbf{n}, R$ is the modulus of the radius vector, and $I_{N}(\mathbf{n})$ is the cubic invariant of rank $N$, which runs over even values, except the value 2 (there is no rank- 2 cubic invariants), i.e., $N=0,4,6$. The actual symmetry of the potential energy of an atom in an octahedral void of fullerite $\mathrm{C}_{60}$ slightly differs from octahedral if the discrete nature of $\mathrm{C}$ atoms on the «surface» of the $\mathrm{C}_{60}$ molecule is taken into account, especially in the orientational glass state. We will ignore this insignificant disturbance; more so, further computations will be based on the generally accepted model of uniform continuous density of carbon atoms on the surface of fullerene molecules using the Lennard-Jones (LJ) [9] potential. The shape of the potential $U(\mathbf{R})$ for a rare gas atom depends essentially on the species. Thus, for the He atom [15], this potential has a bump at $R=0$ and the overall shape resembles a spherical well rather than a parabola. This is because the van der Waals diameter of the He atom is appreciably smaller than the size of the octahedral void. In this paper we chose xenon in order to deal with a potential closer to that of a harmonic oscillator. The final numeric results depend somewhat on the choice of the LJ parameters; those of Vidali and Cole [16] were used in our computations.

To make the task analytically solvable, we introduce the following simplifications, which cannot lead to basically erroneous results. First, we truncate the expansion in Eq. (3), leaving only two terms with $N=0$ and 4. Second, the isotropic potential $V_{0}(R)$ is assumed to be that of a spherical oscillator:

$$
V_{0}(R)=M \omega^{2} R^{2} / 2,
$$

where $\omega$ is the oscillation frequency and $M$ is the dopant mass. This approximation is validated by our calculations which yielded a value of $2.23 \pm 0.04$ for the exponent of $R$ in Eq. (4).

The wave function of the atomic dopant particle, $\Psi$, and the corresponding energy eigen-values are found from the Schrödinger equation:

$-\left(\hbar^{2} / 2 M\right) \Delta \Psi+\left(M \omega^{2} R^{2} / 2\right) \Psi+V_{4}(R) I_{4}(\mathbf{n}) \Psi=E \Psi$, where

$$
I_{4}(\mathbf{n})=\sqrt{7 / 12} Y_{40}(\mathbf{n})+\sqrt{5 / 24}\left(Y_{44}(\mathbf{n})+Y_{4 \overline{4}}(\mathbf{n})\right) .
$$

We note that the sign of this invariant is opposite to that used by Devonshire [11].

The third simplification will be our treating the $N=4$ term as a perturbation. Without the perturbation term, the solutions to this equation for the spherical oscillator, extensively employed in nuclear physics, are well known [17]. We will use the representation of the eigen-functions $\Psi$ in spherical coordinates:

$$
\Psi_{v l m}=F_{v l}(R) Y_{l m}(\mathbf{n}) .
$$

Here $Y_{l m}(\mathbf{n})$ are the spherical harmonics and the radial functions $F_{v l}(R)$ can be represented in the form $\left(r=R(M \omega / \hbar)^{1 / 2}\right)$

$$
F_{v l}(R)=r^{l} \exp \left(-r^{2} / 2\right) \sum_{k=0}^{v} A_{v l k} r^{2 k}
$$

with

$$
A_{v l k}=\frac{(-1)^{k}}{k !}\left[\frac{2(n !)}{\Gamma(v+l+3 / 2)}\right]^{1 / 2} \frac{\Gamma(v+l+3 / 2)}{(n-k) ! \Gamma(k+l+3 / 2)} \text {. }
$$

In Table 1 we give explicit expressions for normalized radial functions $F_{v l}(z)$ represented in the form

$$
f_{v l}(z)=\pi^{1 / 4} \exp \left(z^{2} / 2\right) F_{v l}(R)
$$

Table 1. Quantum numbers and eigen-functions of the spherical oscillator eigen-states

\begin{tabular}{c|c|c|c|c}
\hline \hline$N$ & $v$ & $l$ & Degeneracy & $f_{v l}(z)$ \\
\hline \hline 0 & 0 & 0 & 1 & 2 \\
1 & 0 & 1 & 3 & $\sqrt{8 / 3} z$ \\
2 & 1 & 0 & 1 & $\sqrt{2 / 3}\left(3-2 z^{2}\right)$ \\
& 0 & 2 & 5 & $\sqrt{16 / 15} z^{2}$ \\
3 & 1 & 1 & 3 & $\sqrt{4 / 15}\left(5 z-2 z^{3}\right)$ \\
& 0 & 3 & 7 & $\sqrt{32 / 105} z^{3}$ \\
4 & 2 & 0 & 1 & $\sqrt{1 / 30}\left(15-20 z^{2}+4 z^{4}\right)$ \\
& 1 & 2 & 5 & $\sqrt{8 / 105}\left(7 z^{2}-2 z^{4}\right)$ \\
& 0 & 4 & 9 & $\sqrt{64 / 945} z^{4}$ \\
\hline \hline
\end{tabular}

The energy spectrum is given by

$$
E_{v l}=\hbar \omega(N+3 / 2) \text {. }
$$

with $N=2 v+l=0,1,2, \ldots$ and the total degeneration of level $N$ is

$$
G_{N}=\frac{(N+1)(N+2)}{2} .
$$


To demonstrate the main idea for the case of low temperatures $(T \leq \hbar \omega)$, we calculate the energy for the four lowest states enumerated in Table 1. The radial dependence of $V_{4}(R)$ in Eq. (5) that follows from our computations for $\mathrm{Xe}-\mathrm{C}_{60}$ interaction potential is very close to $R^{4}$; our calculations give $V_{4}(R) \simeq R^{\alpha}, \alpha=4.01 \pm 0.03$. Thus the perturbation term was chosen in the form

$$
V_{4}(R)=\gamma \hbar \omega R^{4}
$$

Applying now the standard procedure by treating the term with $N=4$ as a perturbation up to the second order, we obtain for the energy $E_{p}$ of the state $|p\rangle$ (here the ket vector $|p\rangle$ is a product of the radial and angular ket vectors: $|p\rangle=\mid v, l\}|l, m\rangle)$

$$
E_{p}=E_{p}^{(0)}+V_{p p}+\sum_{q}^{\prime} \frac{\left|V_{p q}\right|^{2}}{E_{p}^{(0)}-E_{q}^{(0)}}
$$

Here $E_{p}^{(0)}$ are the energy eigen-values $\hbar \omega N$ with $N$ as per Table 1 and

$$
V_{p q}=\left\langle p\left|V_{4}(R) I_{4}(n)\right| q\right\rangle .
$$

The energy landscape as a function of the angular variables depends on the sign of the perturbation. Our calculations show that $\gamma$ in Eq. (13) is positive, which means that there are six maxima on the unit sphere along the $(00 \pm 1)$-type axes and eight minima along the (111)-type axes. As the corrugation parameter $\gamma$ increases, the eight wave functions tend to concentrate along the four $(111)-(\overline{1} \overline{1} \overline{1})$ directions; the states in these wells become those of a twodimensional oscillator, and the respective energy vs. $\gamma$ curves cling into ever narrowing bunches. Owing to tunneling through the angular barriers these states sweep over the whole $\theta-\phi$ continuum. The resulting states can be named rotating oscillatory modes. The results of the corresponding calculations of the energy spectrum of a perturbed spherical oscillator as a function of the perturbation parameter are shown in Fig. 1. The main conclusion is that the general view of the spectrum closely reminds that of a symmetric rotor in a potential of cubic symmetry [11].

Transitions within the state of the octet with lowest energies are characterized by negative Grüneisen parameters, and at low enough temperatures the corresponding thermal expansion will be negative. Transitions between any state in the octet to the next-in-energy bunch of levels will produce a characteristic maximum both in thermal expansion and heat capacity.

Detailed calculations for atomic and molecular chemically neutral dopants in $\mathrm{C}_{60}$ are pending.

The authors thank V.G. Manzhelii and A.V. Dolbin for valuable discussions and constructive criticism.

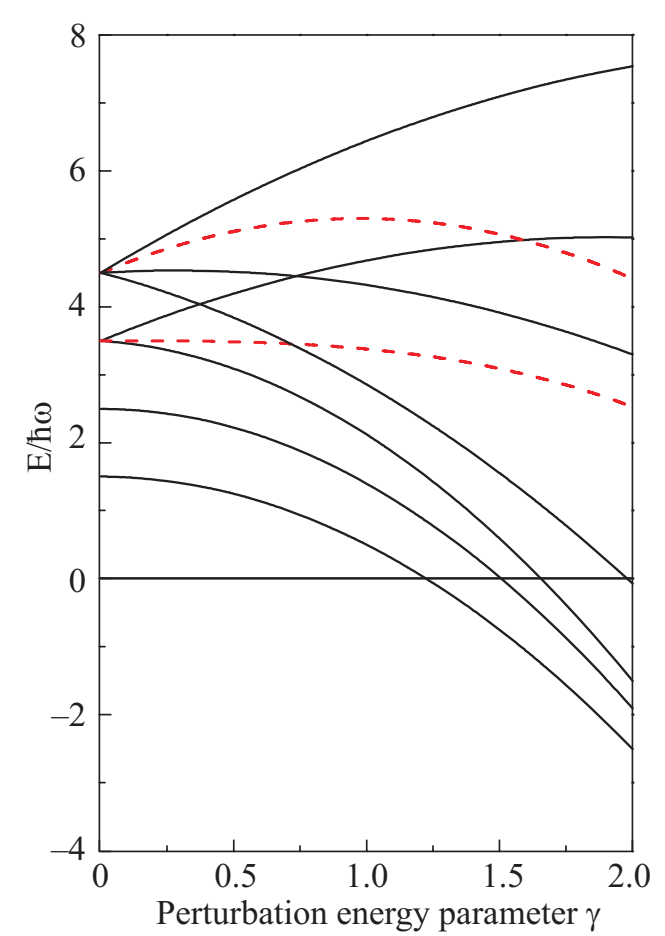

Fig. 1. Energy levels as a function of $\gamma$.

1. F. Gugenberger, R. Heid, C. Meingast, P. Adelmann, M. Brawn, H. Wuhl, M. Haluska, and H. Kuzmany, Phys. Rev. Lett. 69, 3774 (1992).

2. L.S. Fomenko, V.D. Natsik, S.V. Lubenets, V.G. Lirtsman, N.A. Aksenova, A.P. Isakina, A.I. Prokhvatilov, M.A. Strzhemechny, and R.S. Ruoff, Fiz. Nizk. Temp. 21, 465 (1995) [Low Temp. Phys. 21, 364 (1995)].

3. A.N. Aleksandrovskii, V.B. Esel'son, V.G. Manzhelii, B.G. Udovidchenko, A.V. Soldatov, and B. Sundqvist, Fiz. Nizk. Temp. 23, 1256 (1997) [Low Temp. Phys. 23, 943 (1997)].

4. A.N. Aleksandrovskii, A.S. Bakai, A.V. Dolbin, V.B. Esel'son, G.E. Gadd, V.G. Gavrilko, V.G. Manzhelii, S. Moricca, B. Sundqvist, and B.G. Udovidchenko, Fiz. Nizk. Temp. 29, 432 (2003) [ Low Temp. Phys. 29, 324 (2003)].

5. A.N. Aleksandrovskii, A.S. Bakai, D. Cassidy, A.V. Dolbin, V.B. Esel'son, G.E. Gadd, V.G. Gavrilko, V.G. Manzhelii, S. Moricca, and B. Sundqvist, Fiz. Nizk. Temp. 31, 565 (2005) [Low Temp. Phys. 31, 429 (2005)].

6. V.G. Manzhelii, A.V. Dolbin, V.B. Esel'son, V.G. Gavrilko, D. Cassidy, G.E. Gadd, S. Moricca, and B. Sundqvist, Fiz. Nizk. Temp. 32, 913 (2006) [Low Temp. Phys. 32, 695 (2006)].

7. A.V. Dolbin, V.B. Esel'son, V.G. Gavrilko, V.G. Manzhelii, N.A. Vinnikov, G.E. Gadd, S. Moricca, D. Cassidy, and B. Sundqvist, Fiz. Nizk. Temp. 33, 1401 (2007) [Low Temp. Phys. 33, 1068 (2007)].

8. A.V. Dolbin, N.A. Vinnikov, V.G. Gavrilko, V.B. Esel'son, V.G. Manzhelii, and B. Sundqvist, Fiz. Nizk. Temp. 33, 618 (2007) [Low Temp. Phys. 33, 465 (2007)]. 
9. A.N. Aleksandrovskii, K.A. Chishko, V.B. Esel'son, V.G. Manzhelii, and B.G. Udovidchenko, Fiz. Nizk. Temp. 23, 999 (1997) [Low. Temp. Phys. 23, 750 (1997)].

10. Yu.A. Freiman, Fiz. Nizk. Temp. 9, 657 (1983) [Sov. J. Low. Temp. Phys. 9, 335 (1997)].

11. A.F. Devonshire, Proc. Roy. Soc. (London) A153, 601 (1936).

12. V.M. Loktev, J.M. Khalack, and Yu.G. Pogorelov, Fiz. Nizk. Temp. 27, 539 (2001) [ Low Temp. Phys. 27, 397 (2001)].

13. J.M. Khalack and V.M. Loktev, Fiz. Nizk. Temp. 29, 577 (2003) [ Low Temp. Phys. 29, 429 (2003)].
14. V.V. Belotserkovskii and M.A. Strzhemechny, Fiz. Nizk. Temp. 15, 520 (1989) [Sov. J. Low Temp. Phys. 15, 293 (1989)].

15. K.A. Yagotintsev, M.A. Strzhemechny, Yu.E. Stetsenko, I.V. Legchenkova, and A.I. Prokhvatilov, Physica B381, 224 (2006).

16. G. Vidali and M.W. Cole, Phys. Rev. B29, 6736 (1984).

17. M. Moshinsky, The Harmonic Oscillator in Modern Physics: From Atoms to Quarks, Gordon and Breach (1969). 